\title{
Antimicrobial activity of curcumin, cinnamon oil, quercetin and lemon oil
}

\author{
HANR Hapugala ${ }^{1}$, DMRN Dissanayaka ${ }^{1}$, MFF Sazna ${ }^{1}$, BS Nanayakkara ${ }^{2}$, \\ CL Abayasekara ${ }^{2}$, G Pamunuwa $^{1}$
}

Introduction and Objectives: Curcumin, cinnamon oil, quercetin and lemon oil are some plant-derived natural compounds that could be investigated for their antimicrobial potential. The current study was carried out to determine the antimicrobial activity of curcumin, cinnamon oil, quercetin and lemon oil against three bacterial strains (Escherichia coli NCTC 10418, Pseudomonas aeruginosa NCTC 10662, and Staphylococcus aureus NCTC 6571) and one fungus (Candida albicans ATCC 10261).

Methods: The minimum inhibitory concentration (MIC) of each compound against the test microorganisms was investigated using the agar dilution method. Based on the MIC values obtained, the antimicrobial activity of cinnamon oil in combination with curcumin was investigated to ascertain if the effect was synergistic, indifferent or antagonistic by determining the Fractional Inhibitory Concentration Index (FICI).

Results: Cinnamon oil exhibited strong inhibitory activity against all test microorganisms. The MIC of cinnamon oil against E. coli, P. aeruginosa, S. aureus and C. albicans was $1.25 \%(\mathrm{v} / \mathrm{v})$. Curcumin gave the highest antibacterial activity against $S$. aureus compared to the other strains, with an MIC of $0.15 \mathrm{mg} / \mathrm{mL}$. Growth of both Gram-negative bacterial strains, i.e. E. coli and P. aeruginosa was not inhibited at any of the curcumin concentrations tested. The MIC of curcumin against C. albicans was $0.4 \mathrm{mg} / \mathrm{mL}$. Lemon oil $(1.25-10 \%)$ and quercetin $(0.15-0.5 \mathrm{mg} / \mathrm{mL})$ did not inhibit growth of any of the test microorganisms at any of the concentrations tested. There was a synergistic antimicrobial effect between curcumin and cinnamon oil when in combination, against $C$. albicans (FICI $=0.42$ ) while an indifferent activity was found against $S$. aureus $(\mathrm{FICI}=1.12)$.

Conclusions: The antimicrobial activity of cinnamon oil and curcumin warrants their use, either separately or in combination, in medicine and in the food industry.

Keywords: Agar dilution, antibacterial anticandidal, fractional \& minimum inhibitory concentration

\footnotetext{
${ }^{1}$ Department of Horticulture and Landscape Gardening, Faculty of Agriculture and Plantation Management, Wayamba University of Sri Lanka, Makandura, Gonawila, Sri Lanka

${ }^{2}$ Department of Botany, Faculty of Science, University of Peradeniya, Sri Lanka

Address for correspondence: HANR Hapugala. Telephone: +94767039111

Email: nadeeshahapugala@gmail.com (D) https://orcid.org/0000-0003-0329-9645
} 